\title{
Average Thickness of Magnetosheath Upstream of Magnetic Clouds at 1 AU versus Solar Longitude of Source
}

\author{
R.P. Lepping • C.-C. Wu • N. Gopalswamy • \\ D.B. Berdichevsky
}

Received: 25 May 2007 / Accepted: 20 December 2007 / Published online: 1 March 2008

(C) Springer Science+Business Media B.V. 2008

\begin{abstract}
Starting with a large number $(N=100)$ of Wind magnetic clouds (MCs) and applying necessary restrictions, we find a proper set of $N=29$ to investigate the average ecliptic plane projection of the upstream magnetosheath thickness as a function of the longitude of the solar source of the MCs, for those cases of MCs having upstream shock waves. A few of the obvious restrictions on the full set of MCs are the need for there to exist a driven upstream shock wave, knowledge of the MC's solar source, and restriction to only MCs of low axial latitudes. The analysis required splitting this set into two subsets according to average magnetosheath speed: slow/average $\left(300-500 \mathrm{~km} \mathrm{~s}^{-1}\right)$ and fast $\left(500-1100 \mathrm{~km} \mathrm{~s}^{-1}\right)$ speeds. Only the fast set gives plausible results, where the estimated magnetosheath thickness $(\Delta S)$ goes from 0.042 to $0.079 \mathrm{AU}$ (at $1 \mathrm{AU}$ ) over the longitude sector of $0^{\circ}$ (adjusted source-center longitude of the average magnetic cloud) to $40^{\circ}$ off center (East or West), based on $N=11$ appropriate cases. These estimates are well determined with a sigma $(\sigma)$ for the fit of $0.0055 \mathrm{AU}$, where $\sigma$ is effectively the same as $\sqrt{ }$ (chi-squared) for the appropriate quadratic fit. The associated linear correlation coefficient for $\Delta S$ versus |Longitude| was very good (c.c. $=0.93$ ) for the fast range, and $\Delta S$ at $60^{\circ}$ longitude is extrapolated to be 2.7 times the value at $0^{\circ}$. For the slower speeds we obtain the surprising result that $\Delta S$ is typically more-or-less constant at $0.040 \pm 0.013 \mathrm{AU}$ at all longitudes, indicating that the $\mathrm{MC}$ as a driver, when moving close to the normal solar wind speed, has little influence on magnetosheath thickness. In some cases, the correct choice between two candidate solarsource longitudes for a fast MC might be made by noting the value of the observed $\Delta S$ just
\end{abstract}

\footnotetext{
R.P. Lepping $(\bowtie)$

Space Weather Laboratory, NASA/GSFC, Greenbelt, MD 20771, USA

e-mail: ronald.p.lepping@ nasa.gov

C.-C. Wu

University of Alabama in Huntsville, Huntsville, AL 35899, USA

N. Gopalswamy

Solar System Exploration Division, NASA/GSFC, Greenbelt, MD 20771, USA

D.B. Berdichevsky

Sigma Space Corporation, 4801 Forbes Boulevard, Lanham, MD 20706, USA
} 
upstream of the MC. Also, we point out that, for the 29 events, the average sheath speed was well correlated with the quantity $\Delta V\left[=\left(\left\langle V_{\mathrm{MC}}\right\rangle-\left\langle V_{\text {UPSTREAM }}\right\rangle\right)\right]$, and also with both $\left\langle V_{\mathrm{MC}}\right\rangle$ and $\left\langle V_{\mathrm{MC}, \mathrm{T}}\right\rangle$, where $\left\langle V_{\mathrm{MC}}\right\rangle$ is the first one-hour average of the MC speed, $\left\langle V_{\mathrm{MC}, \mathrm{T}}\right\rangle$ is the average MC speed across the full MC, and $\left\langle V_{\text {UPSTREAM }}\right\rangle$ is a five-hour average of the solar wind speed just upstream of the shock.

Keywords Magnetic clouds · Solar wind · Shock waves · Magnetosheath

\section{Introduction}

We are concerned with estimating the thickness, in the ecliptic plane at $1 \mathrm{AU}$, of the magnetosheath formed upstream of those magnetic clouds (MCs) that drive interplanetary shock waves, as a function of the solar longitude of the source of the MC. A MC is defined as a region in the solar wind having (1) enhanced magnetic field strength, (2) a smooth change in field direction as observed by a spacecraft passing through the $\mathrm{MC}$, and (3) low proton temperature (and low proton plasma beta) compared to the ambient proton temperature; see Burlaga et al. (1981), Klein and Burlaga (1982), Burlaga (1988, 1995), and Lepping and Berdichevsky (2000) for the definition of a MC and for discussions of its properties and some of its effects at Earth and those expected at other planets. The magnetosheath originates because the driver (i.e., the MC) is moving faster than the fast mode MHD wave in the local upstream medium, so a shock arises. The magnetosheath is just the turbulent region bounded by the shock ramp on the one side and the front surface of the driver on the other; see Russell and Mulligan (2002a), who study magnetosheaths upstream of solar ejecta, partly to infer the cross-sectional geometry of the ejecta.

In Figure 1 (ecliptic plane view) we show a sketch of how we expect the magnetosheath thickness $(\Delta S)$ to vary as a function of ecliptic-plane longitude, being small, medium, or large depending on how far, longitudinally, the observer is located with respect to the MC's (assumed curved) center line, which starts at the solar source. The notion of this kind of geometry for the Earth's magnetosheath region arises from the fact that the global locus of the ramp of the upstream hydromagnetic shock wave should have a cross-sectional shape in the ecliptic plane approximating a hyperbola (e.g., Spreiter, Summers, and Alksne, 1966), whereas the MC's front boundary's cross section should be confined to a more restricted region, being closer to an ellipse (or perhaps, on occasion, a circle) (e.g., Fairfield, 1971). For the cases of ejecta-related sheaths, Russell and Mulligan (2002a, 2002b) studied the shock standoff distance, which is known to depend on the shape of the shock driver, and point out that the thicknesses of the solar-ejecta sheaths generally appear too large to be consistent with ejecta of circular cross-sectional shape. The authors suggest that the radial (with respect to the Sun's position) thickness is smaller than the other two dimensions. These studies were based on two spacecraft, ISEE-3 and PVO. However, since we have not yet had the necessary fleet of spacecraft in all of the right places to test accurately the degree of a noncircularily, nor to test the idea of a variable magnetosheath thickness (of this specific type) for any given $\mathrm{MC}$, we choose to make such a study on average, starting with a large number of cases of MCs from a single spacecraft. As we will see, various necessary restrictions will reduce the reliability of the result, because of the much smaller number of cases that remain after satisfying these restrictions. Such restrictions include the following: the need to know the solar source for a given MC, the constraint that the source be close to the solar equator, the need for the existence of a shock upstream of the $\mathrm{MC}$, the restriction that the MC have an axis that is in or near the ecliptic plane and that it be of sufficiently 


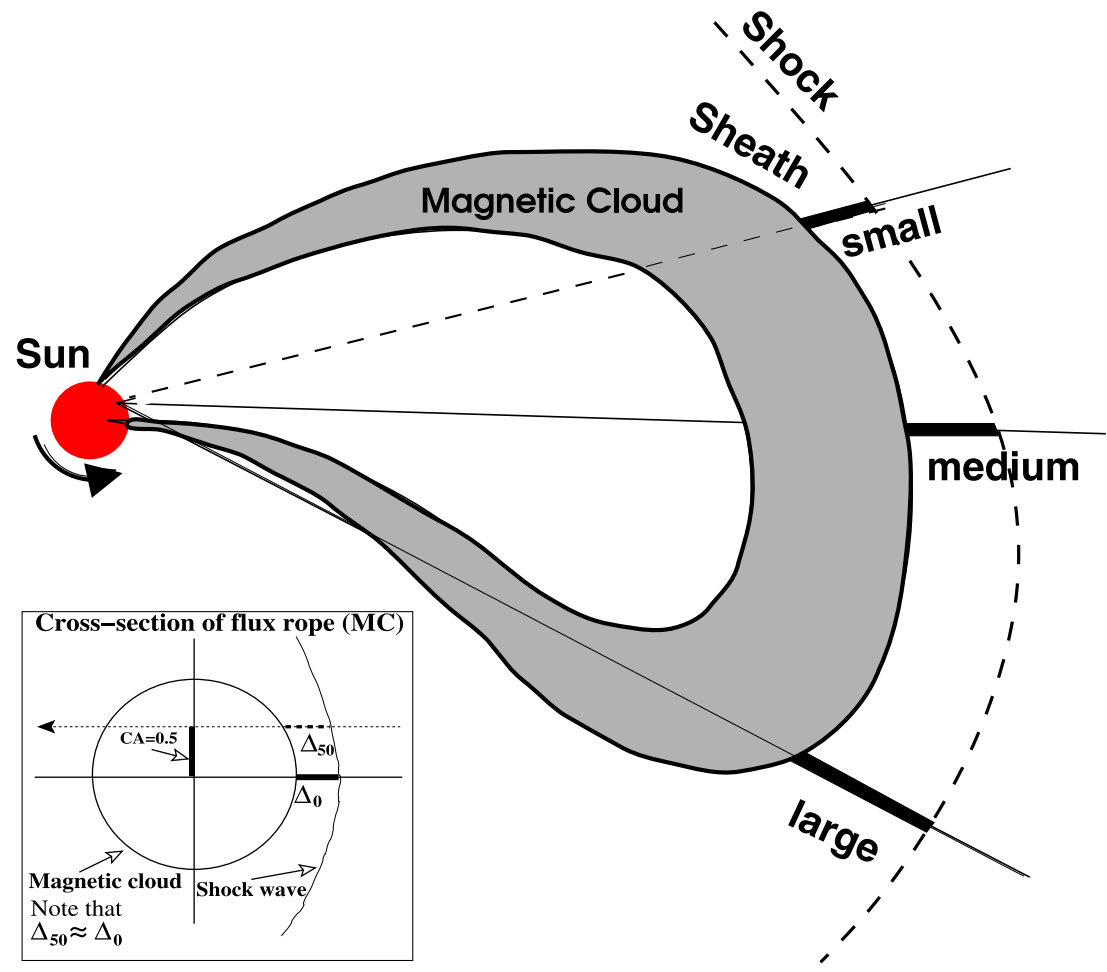

Figure 1 A sketch of a global MC, from Marubashi (1986, 1997), as a driver of an upstream shock wave where the change in thickness of the upstream magnetosheath according to longitude is accentuated. The inset shows the cross-sectional view, where the radius of curvature of the MC's front surface is assumed approximately that of the upstream shock surface near the ecliptic plane. The dashed line is the relative spacecraft trajectory, assumed here to be parallel to the ecliptic plane. The subscript on $\Delta$ (e.g., $\left.\Delta_{50}\right)$ refers to the closest approach distance in terms of the radius of the $\mathrm{MC}$, in percentage.

long duration (to avoid atypically small cases), and the need to have intermediate or smaller closest approach distance of the spacecraft's path to the MC's axis, for reliability. These limitations eliminate a large percentage of MCs. Nevertheless, we start with a such a large number of MCs that it is believed that the final result is statistically significant.

MCs or any relatively fast solar ejecta often drive upstream interplanetary shocks and therefore possess upstream magnetosheaths (henceforth called sheaths). Such sheath regions are of concern for many reasons, two of which are (1) the effect that a sheath usually has when encountering and interacting with Earth's magnetosphere (see, e.g., Gosling and McComas, 1987, and McComas et al., 1988, both of which discuss the possibility of strong southward draping of the IMF in the region just upstream of fast solar ejecta and the expected resulting geomagnetic activity) and (2) the sheath's ability to act as a barrier to cosmic rays, causing a Forbush decrease (e.g., see Morrison, 1956; Badruddin, Venkatesan, and Zhu, 1991; Lepping et al., 1991). The first is related to the speed of the passing sheath, as well as to intrinsic properties of the sheath itself such as the intensity and structure of its magnetic field (i.e., the direction of the field and length of time it keeps a particular direction, especially "southward" in a Geocentric Solar Magnetospheric coordinate system), and the second is related to the sheath's turbulent nature (e.g., see Morrison, 1956). See Owens et al. (2005) and Liu et al. (2006a) for discussions 
on the characteristics of sheath regions ahead of ejecta, referred to as interplanetary coronal mass ejections (ICMEs) in their cases. Specifically, in the former case the authors studied the possibility of predicting the average field intensity within such sheath regions based on an empirical relationship (that they developed) requiring measurements of the upstream solar wind speed and the so-called local ICME cruise speed. This analysis was done in the hope of using such predicted fields to help in forecasting the geomagnetic effects resulting from such a sheath encountering the Earth. Liu et al. (2006a) carried out a superposed epoch analysis of plasma and magnetic field data from ACE and Wind spacecraft in the study of the plasma depletion layer (and associated mirror mode waves) ahead of ICMEs, with and without upstream shocks, and discuss the implications. They also point out that the plasma depletion layer of a MC is expected to coincide with magnetic field draping around the MC.

The basic characteristics of interplanetary MCs are well known, and a more subtle understanding of them is growing rapidly, but less appears to be known about the sheath regions that often exist in front of them (i.e., when the MCs drive upstream shock waves creating the sheaths). The situation between the driver (the MC) and the upstream shock wave is analogous to the Earth's magnetosphere (the "driver") and Earth's bow shock, and similarly for other planets (see, e.g., Spreiter, 1975, for the terrestrial planets). One important difference that should be pointed out, however, is that the relative speed between the solar wind and a planetary magnetosphere is relatively high (on average being about $420 \mathrm{~km} \mathrm{~s}^{-1}$ at Earth, for example; see Wolfe, 1972), but the relative speed between a MC and the upstream solar wind is much slower, (i.e., usually lower by about an order of magnitude or so), resulting in weak or intermediate strength shocks, compared to the strong shocks at Earth and the other planets. Nevertheless, a MC can be thought of as a huge magnetosphere, but perhaps strangely shaped, moving out into the solar wind at a speed close to but often faster than the solar wind at $1 \mathrm{AU}$, and often driving a shock wave, depending on upstream conditions. Apparently the solar wind is partly responsible for convecting the MC outward (Burlaga, 1995), along with the consequences of the thrust of its initial solar eruption. Hence, it should not be surprising that many MCs are moving at speeds close to, but slightly faster than, the local solar wind speed at $1 \mathrm{AU}$.

\section{Data Employed}

The MCs of concern here were originally taken from the list of Wind MCs given at the NASA Space Flight Center Wind/MFI Web site http://lepmfi.gsfc.nasa.gov/mfi/mag_cloud_ S1.html, where cases from early 1995 to mid-April $2006(N=100 \mathrm{MCs})$ are provided along with their modeled properties, derived from the Lepping, Jones, and Burlaga (1990) MC parameter fitting model. Since we must start at 24 December 1996 (explained in the following), this reduces the list to $N=83 \mathrm{MCs}$. Then we retain only those cases that satisfy two constraints: (1) A reliable solar source must exist from the list provided by Gopalswamy et al. (2007) and (2) the MC must have an upstream shock. [Typically about three-quarters of all MCs at 1 AU (Lepping et al., 2002) are observed to have upstream shocks or shock-like structures.] This reduces the cases of acceptable MCs to $N=58$. Since we are constraining the results for application to the ecliptic plane, two other restrictions were applied: (1) The latitude of the solar source $\left(\theta_{\mathrm{S}}\right)$ had to be within $30^{\circ}$ of the Sun's equator $\left(\right.$ i.e., $<30^{\circ} \mathrm{N}$ or $<30^{\circ} \mathrm{S}$, or $\left|\theta_{\mathrm{S}}\right|<30^{\circ}$ ), giving $N=51 \mathrm{MCs}$, and (2) the model estimated latitude $\left(\theta_{\mathrm{A}}\right)$ of the $\mathrm{MC}$ axis had to be $40^{\circ}$ or less (i.e., $\left|\theta_{\mathrm{A}}\right| \leq 40^{\circ}$ ), reducing acceptable cases to $N=36$.

Finally, we restrict the acceptable cases to those where the closest approach of the spacecraft to the $\mathrm{MC}$ axis was not excessive, so that the ratio $\left|Y_{\mathrm{O}} / R_{\mathrm{O}}\right|$ must be 0.80 or smaller, 
where $Y_{\mathrm{O}}$ is the coordinate of the closest approach distance and $R_{\mathrm{O}}$ is the radius of the MC, both estimated by the Lepping, Jones, and Burlaga (1990) model and given on the Wind/MFI Web site. This eliminates six more cases, and another case is eliminated, because it was unusually short in duration (6.5 hours), giving a final set of MCs for our analysis of $N=29$. This is referred to as the "reduced" set of MCs, and all further analysis is carried out with this reduced set. Finally, working with a rather small number of MCs in this reduced set forces us to assume that $\Delta S$ is axially symmetric in longitude about the MC's "central line" to a first approximation and fold all West and East events together. Under the column "Start time" in Table 1 we give the times of the 29 MCs in the reduced set; the splitting by MC speed is explained in the following.

\section{Analysis and Findings}

\subsection{Average Speeds of Sheaths and Magnetic Clouds}

Figure 2A shows a plot of $\left\langle V_{\text {SHEATH }}\right\rangle$ versus $\left\langle V_{\text {MC,T }}\right\rangle$ for the $N=29 \mathrm{MCs}$ of the reduced set, where $\left\langle V_{\text {SHEATH }}\right\rangle$ is the average speed of the sheath plasma and $\left\langle V_{\mathrm{MC}, \mathrm{T}}\right\rangle$ is the average MC speed taken across the full MC. Notice that the linear correlation coefficient (c.c. $=0.84$, shown in the figure) is quite good. If we consider the average across all of these events for both $\left\langle V_{\text {SHEATH }}\right\rangle$ and $\left\langle V_{\text {MC,T }}\right\rangle$ we obtain $\left\langle\left\langle V_{\text {SHEATH }}\right\rangle\right\rangle=510 \mathrm{~km} \mathrm{~s}^{-1}$ and $\left\langle\left\langle V_{\text {MC,T }}\right\rangle\right\rangle=$ $481 \mathrm{~km} \mathrm{~s}^{-1}$, giving a percent difference of only $6.0 \%$. It is interesting that if one considers only the slower cases, that is, those in the range of $300-500 \mathrm{~km} \mathrm{~s}^{-1}$ (considering sheath speeds) where $N=17$, this percent difference is only $1.9 \%$, showing that the shocked solar wind speed in the sheath is, on average, hardly altered at all by the driver (the MC) for the slower cases. In contradistinction, if only the faster cases are considered (where $N=12$ ), that is, those with sheath speeds in the range of $500-1100 \mathrm{~km} \mathrm{~s}^{-1}$, we obtain a percent difference in speed of $10.3 \%$, a significant difference.

In Figure $2 \mathrm{~B}$ we show a similar plot, that of $\left\langle V_{\text {SHEATH }}\right\rangle$ versus $\langle\Delta V\rangle$, where $\Delta V$ is given by $\left(\left\langle V_{\mathrm{MC}}\right\rangle-\left\langle V_{\text {UPSTREAM }}\right\rangle\right),\left\langle V_{\mathrm{MC}}\right\rangle$ is the average of the plasma speed for the first one hour within the $\mathrm{MC}$, and $\left\langle V_{\mathrm{UPSTREAM}}\right\rangle$ is a five-hour average speed of the solar wind immediately upstream of the shock. (In actuality, the interval for the average solar wind speed $\left\langle V_{\text {UPSTREAM }}\right\rangle$ was separated from the upstream shock by 12 minutes, to avoid any possible precursor-shock fluctuations.) The c.c., 0.80 for this case, is relatively good, similar to that for the plot of $\left\langle V_{\text {SHEATH }}\right\rangle$ versus $\left\langle V_{\mathrm{MC}, \mathrm{T}}\right\rangle$. It is interesting that the c.c. of panel A of Figure 2 (0.084) is slightly higher than that of panel B. In both panels a straight-line fit to the data is shown. A third correlation was attempted, that of $\left\langle V_{\text {SHEATH }}\right\rangle$ versus $\left\langle V_{\mathrm{MC}}\right\rangle$ (for the first hour of the MC) and, as might be expected, the c.c. was very high, 0.96 (not shown). This is apparently because the MC's front boundary, which is usually a tangential discontinuity (e.g., see Lepping, Wu, and Berdichevsky, 2005), is expected to have the same speed immediately on both sides, and generally $\left\langle V_{\text {SHEATH }}\right\rangle$, averaged across the full sheath, is close to the sheath's speed near the MC's front boundary.

\subsection{Sheath Thickness and Assumptions}

Here we describe the formalism and the assumptions that are employed in estimating sheath thickness $(\Delta S)$. We assume the simple relationship that

$$
\Delta S=\left\langle V_{\mathrm{SHEATH}}\right\rangle \Delta t
$$


Table 1 Characteristics of reduced set of magnetic clouds and sheath speeds.

\begin{tabular}{|c|c|c|c|c|c|c|c|c|}
\hline $\begin{array}{l}\text { Code } \\
\text { no. }\end{array}$ & $\begin{array}{l}\text { Start time } \\
\text { (Year/DOY, hr) }\end{array}$ & $\begin{array}{l}\text { Duration } \\
\text { (hr) }\end{array}$ & $\left(\theta_{\mathrm{A}}, \phi_{\mathrm{A}}\right)^{\mathrm{a}}$ & $\begin{array}{l}\text { Diameter } \\
(\mathrm{AU})\end{array}$ & $\left|Y_{\mathrm{O}} / R_{\mathrm{O}}\right|$ & $\begin{array}{l}\text { MC speed } \\
\left(\mathrm{km} \mathrm{s}^{-1}\right)\end{array}$ & $\begin{array}{l}\text { Sheath speed } \\
\left(\mathrm{km} \mathrm{s}^{-1}\right)\end{array}$ & $Q_{\mathrm{O}}$ \\
\hline \multicolumn{9}{|c|}{ Slow/average sheath speed range: $300-500 \mathrm{~km} \mathrm{~s}^{-1}, N=17$} \\
\hline 11 & $1996 / 359,02.8$ & 32.5 & $\left(26^{\circ}, 082^{\circ}\right)$ & 0.286 & 0.47 & 355 & 413.5 & 1 \\
\hline 12 & $1997 / 010,05.3$ & 21.0 & $\left(1^{\circ}, 247^{\circ}\right)$ & 0.190 & 0.11 & 436 & 444.1 & 1 \\
\hline 15 & $1997 / 135,09.1$ & 16.0 & $\left(0^{\circ}, 108^{\circ}\right)$ & 0.186 & 0.24 & 450 & 422.1 & 2 \\
\hline 23 & $1997 / 274,16.3$ & 30.5 & $\left(34^{\circ}, 138^{\circ}\right)$ & 0.395 & 0.44 & 450 & 459.3 & 2 \\
\hline 25 & $1997 / 311,15.8$ & 12.5 & $\left(38^{\circ}, 244^{\circ}\right)$ & 0.220 & 0.16 & 440 & 455.9 & 2 \\
\hline 31 & $1998 / 063,14.3$ & 40.0 & $\left(21^{\circ}, 121^{\circ}\right)$ & 0.327 & 0.06 & 360 & 385.9 & 1 \\
\hline 47 & 2000/210, 21.1 & 13.0 & $\left(-12^{\circ}, 274^{\circ}\right)$ & 0.229 & 0.53 & 471 & 451.0 & 2 \\
\hline 51 & 2000/277, 17.1 & 21.0 & $\left(33^{\circ}, 058^{\circ}\right)$ & 0.184 & 0.23 & 409 & 440.1 & 1 \\
\hline 52 & $2000 / 287,18.4$ & 22.5 & $\left(-37^{\circ}, 146^{\circ}\right)$ & 0.238 & 0.11 & 395 & 449.7 & 2 \\
\hline 53 & $2000 / 302,18.4$ & 23.3 & $\left(26^{\circ}, 089^{\circ}\right)$ & 0.536 & 0.20 & 375 & 383.5 & 3 \\
\hline 55.1 & $2001 / 078,23.3$ & 19.0 & $\left(-34^{\circ}, 213^{\circ}\right)$ & 0.165 & 0.19 & 420 & 430.9 & 1 \\
\hline 62 & $2001 / 304,21.3$ & 37.0 & $\left(-3^{\circ}, 078^{\circ}\right)$ & 0.281 & 0.09 & 340 & 375.5 & 3 \\
\hline 64 & 2001/078, 22.9 & 16.5 & $\left(20^{\circ}, 045^{\circ}\right)$ & 0.207 & 0.18 & 370 & 406.1 & 2 \\
\hline 65 & 2002/083, 03.8 & 43.0 & $\left(35^{\circ}, 288^{\circ}\right)$ & 0.431 & 0.08 & 440 & 465.0 & 2 \\
\hline 72.2 & $2002 / 273,22.6$ & 13.3 & $\left(-16^{\circ}, 110^{\circ}\right)$ & 0.133 & 0.10 & 381 & 351.7 & 3 \\
\hline 79 & $2004 / 204,15.4$ & 07.8 & $\left(-26^{\circ}, 067^{\circ}\right)$ & 0.097 & 0.18 & 500 & 474.3 & 3 \\
\hline 81 & $2004 / 242,18.7$ & 26.0 & $\left(-8^{\circ}, 054^{\circ}\right)$ & 0.240 & 0.06 & 393 & 423.3 & 1 \\
\hline \multicolumn{9}{|c|}{ Fast sheath speed range: $500-1100 \mathrm{~km} \mathrm{~s}^{-1}, N=12$} \\
\hline 43 & $2000 / 043,17.1$ & 07.8 & $\left(-17^{\circ}, 200^{\circ}\right)$ & 0.077 & 0.33 & 543 & 567.2 & 3 \\
\hline 49 & $2000 / 225,06.1$ & 23.0 & $\left(3^{\circ}, 095^{\circ}\right)$ & 0.284 & 0.01 & 567 & 611.7 & 2 \\
\hline 54 & $2000 / 311,23.1$ & 19.0 & $\left(-9^{\circ}, 114^{\circ}\right)$ & 0.276 & 0.19 & 535 & 595.2 & 2 \\
\hline 57 & 2001/102, 07.9 & 10.0 & $\left(31^{\circ}, 205^{\circ}\right)$ & 0.249 & 0.68 & 670 & 696.9 & 2 \\
\hline 59 & $2001 / 119,01.9$ & 11.0 & $\left(31^{\circ}, 119^{\circ}\right)$ & 0.232 & 0.39 & 640 & 690.7 & 2 \\
\hline 60 & $2001 / 148,11.9$ & 22.5 & $\left(-12^{\circ}, 049^{\circ}\right)$ & 0.251 & 0.37 & 475 & 560.5 & 1 \\
\hline 63 & $2001 / 328,15.8$ & 21.5 & $\left(31^{\circ}, 103^{\circ}\right)$ & 0.561 & 0.80 & 730 & 1080.5 & 3 \\
\hline 66 & $2002 / 108,04.3$ & 22.0 & $\left(-27^{\circ}, 318^{\circ}\right)$ & 0.317 & 0.53 & 480 & 537.9 & 1 \\
\hline 67 & $2002 / 110,11.8$ & 29.0 & $\left(24^{\circ}, 156^{\circ}\right)$ & 0.289 & 0.44 & 510 & 580.1 & 3 \\
\hline 71 & $2002 / 214,07.4$ & 13.8 & $\left(-9^{\circ}, 245^{\circ}\right)$ & 0.254 & 0.11 & 493 & 502.7 & 2 \\
\hline 80 & 2004/206, 12.8 & 24.5 & $\left(-21^{\circ}, 086^{\circ}\right)$ & 0.355 & 0.30 & 573 & 590.3 & 2 \\
\hline 82 & $2004 / 313,03.4$ & 13.3 & $\left(-5^{\circ}, 047^{\circ}\right)$ & 0.173 & 0.27 & 674 & 558.5 & 2 \\
\hline
\end{tabular}

${ }^{\mathrm{a}}$ The quantities $\theta_{\mathrm{A}}$ and $\phi_{\mathrm{A}}$ are the latitude and longitude, respectively, of the MC axis in Geocentric Solar Ecliptic coordinates, as estimated by the MC model of Lepping, Jones, and Burlaga (1990).

${ }^{b}$ The MC speed is from an average taken across the MC's full duration.

where $\Delta t=t_{\mathrm{MC}}-t_{\mathrm{S}}$, and where $t_{\mathrm{MC}}$ is the crossing time of the MC's front boundary, $t_{\mathrm{S}}$ is the crossing time of the upstream shock ramp, and $\left\langle V_{\text {SHEATH }}\right\rangle$ is the average speed of the sheath plasma. Most of the chosen MCs are of good quality, with $Q_{\mathrm{O}}=1,2$ (where $Q_{\mathrm{O}}=3$ is poor). Briefly, $Q_{\mathrm{O}}$ depends on the following MC parameters: the chi-squared value of the fit, a comparison of two independent means of estimating the MC's radius where only one means depends on duration, the value of closest approach distance, reasonableness of 

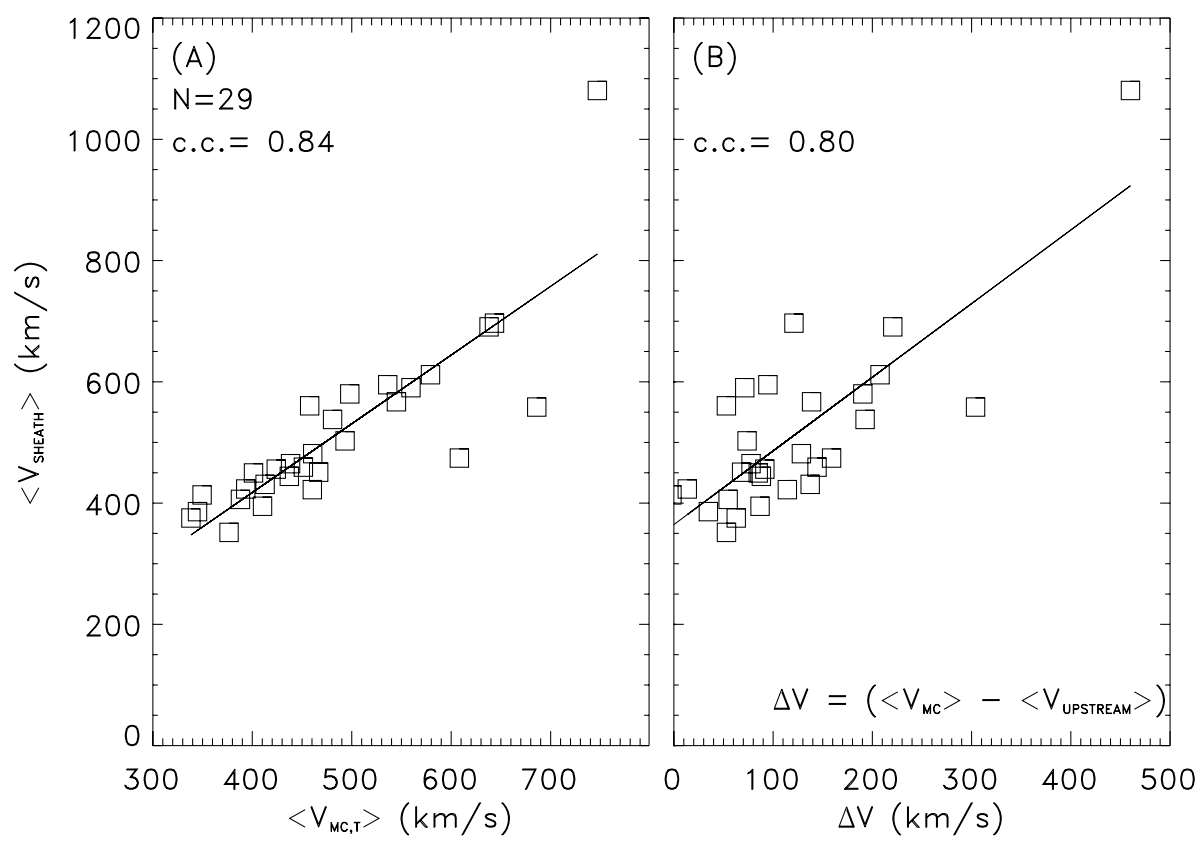

Figure 2 (A) A scatter plot of the average sheath speed $\left\langle V_{\text {SHEATH }}\right\rangle$ versus $\left\langle V_{\mathrm{MC}, \mathrm{T}}\right\rangle$ (averaged over the full duration of the MC) for the reduced set of MCs $(N=29)$ showing how well correlated the average sheath and MC speeds are for this set generally. As shown, the linear correlation coefficient (c.c.) is 0.84 . (B) A scatter plot of $\left\langle V_{\text {SHEATH }}\right\rangle$ versus $\Delta V$, defined as the difference between the average MC speed $\left\langle V_{\text {MC }}\right\rangle$ (now averaged over only the first hour of the MC) and a five-hour average speed of the solar wind immediately upstream of the shock, as defined in panel B. The correlation coefficient (c.c.) was a relatively good 0.80.

the estimated diameter, reasonableness of profile symmetry, comparison of the MC axis alignment to what an axis in the MC's flanks (viewed globally) would be, and a check of the sign/strength of the axial-field component in a specific MC coordinate system. (See Appendix A of Lepping et al., 2006, for a rigorous definition of $Q_{\mathrm{O}}$.) Also it was required that no $\mathrm{MC}$ have a closest approach value greater than $|\mathrm{CA}|\left(\equiv\left|Y_{\mathrm{O}}\right| / R_{\mathrm{O}}\right)=0.8$, where $\left|Y_{\mathrm{O}}\right|$ is the closest approach distance, and $R_{\mathrm{O}}$ is the MC's radius, which could be considered an average radius if the MC has a elliptical (or similar) cross section, provided $R$ (major) and $R$ (minor) are not severely different. See the inset of Figure 1, which shows (for an assumed approximately circular cross-sectional flux rope $\mathrm{MC}$ ) that, under our assumptions, it follows that $\Delta_{50}$ (fixed longitude) $\approx \Delta_{0}$ (fixed longitude), where here fixed longitude refers to any meridian measured with respect to the center of the flux rope and the subscript refers to the $\mathrm{CA}$ value in percentage. This means that in this restricted range of distance normal to the ecliptic plane we assume that the curvature of the front MC surface and that of the driven upstream shock wave surface are approximately the same, and nearly circular for the shock surface. In fact, it is likely that the MC has an elongated cross-sectional shape with the long axis approximately perpendicular to the ecliptic plane, as suggested by Riley and Crooker (2004); also see Liu et al. (2006b). If this is true, then the shock surface and MC front surface may be nearly parallel for a considerable range of CAs. In that case, for example, even $\Delta_{70}$ (fixed longitude) $\approx \Delta_{0}$ (fixed longitude). Hence, for reasonable assumptions, we conclude that, as long as the $\mathrm{CA}$ is not too large, $\Delta S$ in Equation (1) apparently depends mainly on longitudinal changes and not latitudinal ones. We choose MCs that satisfy this 
assumption, because we wish to examine only the longitudinal change in $\Delta S$ around an average $\mathrm{MC}$, the ecliptic plane view. Note that we could have just as easily defined $\Delta S$ as $\Delta S=V_{\mathrm{MC}} \Delta t$ (or even as $\Delta S=V_{\mathrm{MC}, \mathrm{T}} \Delta t$ ), where $V_{\mathrm{MC}}$ is the front speed of the MC of interest, and, as before, $\Delta t=t_{\mathrm{MC}}-t_{\mathrm{S}}$, but our results (i.e., $\Delta S$ versus |Longitude|) would not have been altered significantly because of the good correlation between $\left\langle V_{\text {SHEATH }}\right\rangle$ and $\left\langle V_{\mathrm{MC}}\right\rangle$, with a c.c. of 0.96 (and between $\left\langle V_{\mathrm{SHEATH}}\right\rangle$ and $\left\langle V_{\mathrm{MC}, \mathrm{T}}\right\rangle$, with a c.c. of 0.84 , as shown in Figure 2A).

\section{3. $\Delta S$ versus Solar Longitude}

The solar sources of the MCs were determined and presented by Gopalswamy et al. (2007). The determinations were made in the following way. Guided by the timing of the subsequent MC events (as observed mainly by Wind), locations of the most likely associated H $\alpha$ or soft $\mathrm{X}$-ray flares, as listed in the on-line Solar Geophysical Data, were acquired. If the source locations were not listed, the authors used SOHO EUV movies, soft X-ray data from Yohkoh, or microwave images from the Nobeyama radio-heliograph. The attempt was to identify the MC-associated solar eruption from one or more of the following signatures: coronal dimming, filament eruption, and post-eruption arcade formation.

It was immediately evident that markedly different sheath thickness results would be found when the reduced set of MCs is examined according to their average sheath speeds, that is, whether they are slow/average $\left(300-500 \mathrm{~km} \mathrm{~s}^{-1}\right)$ or fast $\left(500-1100 \mathrm{~km} \mathrm{~s}^{-1}\right)$, so we split the set up according to these speed ranges. Table 1 shows the reduced set of MCs separated according to these speed ranges, and given in terms of code number (as provided by the Wind/MFI Web site), MC start time, duration, axial latitude and longitude $\left(\theta_{\mathrm{A}}, \varphi_{\mathrm{A}}\right)$, diameter, $|\mathrm{CA}|=\left|Y_{\mathrm{O}} / R_{\mathrm{O}}\right|$, MC speed, sheath speed, and MC fit quality (meaning $Q_{\mathrm{O}}=$ 1,2 , or 3 )

Figure 3 shows $\Delta S$ versus the absolute value of the longitude of the solar source of the MCs from the slow/average part of the reduced set. Before the data are plotted in Fig-
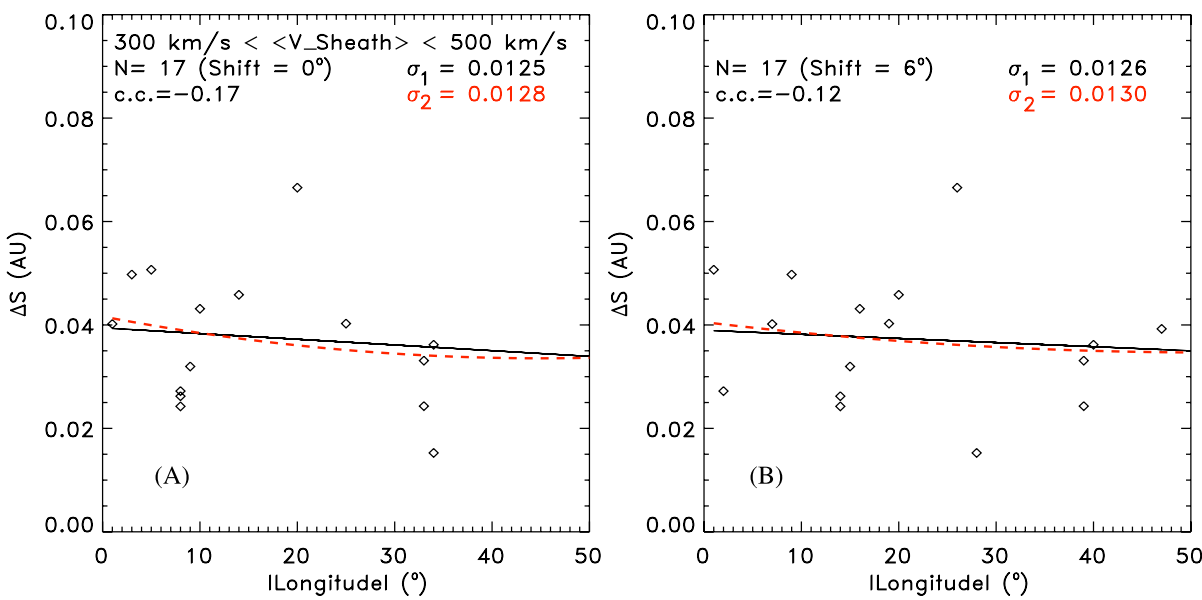

Figure 3 Sheath thickness $(\Delta S)$ versus the absolute magnitude of the longitude of the solar source (|Longitude $\mid$ ) of the magnetic clouds from the reduced set of MCs for the slow/average sheath speed range (i.e., $300-500 \mathrm{~km} \mathrm{~s}^{-1}$ ), where the number of cases is $N=17$ (Table 1, top); the actual observations are given as diamonds. (A) and (B) provide the least-squares fits for $0^{\circ}$ and $6^{\circ}$ longitude shifts, respectively. The quadratic least-squares fits are given as red dashed curves and the straight-line fits are given as black lines. 
Table 2 Straight-line and quadratic fitting results.

\begin{tabular}{lllllllr}
\hline \multicolumn{9}{l}{$\begin{array}{l}\text { Straight-line fit } \\
\text { a }\end{array}$} \\
$\begin{array}{lllllll}\text { Fig. } \\
\text { no. }\end{array}$ & $N$ & $\begin{array}{l}\text { Speed } \\
\text { range }\end{array}$ & $\Delta$ long. $^{\text {b }}$ & c.c. & $\begin{array}{l}\text { Sigma }^{\mathrm{c}} \\
(\mathrm{AU})\end{array}$ & $\begin{array}{l}a \\
\text { (AU) }\end{array}$ & \multicolumn{1}{l}{$\begin{array}{l}b \\
\text { (AU/deg) }\end{array}$} \\
\hline 3A & 17 & Slow & $0^{\circ}$ & -0.17 & 0.0125 & 0.0394 & -0.000110 \\
3B & 17 & Slow & $6^{\circ}$ & -0.12 & 0.0126 & 0.0390 & -0.000080 \\
4A & 12 & Fast & $0^{\circ}$ & 0.77 & 0.0108 & 0.0398 & 0.000945 \\
4B & 12 & Fast & $6^{\circ}$ & 0.81 & 0.0099 & 0.0364 & 0.000964 \\
4C & 11 & Fast & $0^{\circ}$ & 0.86 & 0.0082 & 0.0421 & 0.000922 \\
4D & 11 & Fast & $6^{\circ}$ & 0.93 & 0.0057 & 0.0385 & 0.000975
\end{tabular}

Quadratic curve fit ${ }^{\mathrm{d}}$

\begin{tabular}{|c|c|c|c|c|c|c|c|c|c|c|c|}
\hline $\begin{array}{l}\text { Fig. } \\
\text { no. }\end{array}$ & $N$ & $\begin{array}{l}\text { Speed } \\
\text { range }\end{array}$ & $\Delta$ long. ${ }^{b}$ & $\begin{array}{l}\text { Sigma }^{\mathrm{c}} \\
(\mathrm{AU})\end{array}$ & $\begin{array}{l}a^{\prime} \\
(\mathrm{AU})\end{array}$ & $\begin{array}{l}b^{\prime} \\
(\mathrm{AU} / \mathrm{deg})\end{array}$ & $\begin{array}{l}c^{\prime} \\
\left(\mathrm{AU} / \mathrm{deg}^{2}\right)\end{array}$ & $\begin{array}{l}\Delta S\left(0^{\circ}\right) \\
(\mathrm{AU})\end{array}$ & $\begin{array}{l}\Delta S\left(10^{\circ}\right) \\
(\mathrm{AU})\end{array}$ & $\begin{array}{l}\Delta S\left(20^{\circ}\right) \\
(\mathrm{AU})\end{array}$ & $\begin{array}{l}\Delta S\left(40^{\circ}\right) \\
(\mathrm{AU})\end{array}$ \\
\hline $3 \mathrm{~A}$ & 17 & Slow & $0^{\circ}$ & 0.0128 & 0.0417 & -0.00037 & 0.000004 & 0.042 & 0.038 & 0.036 & 0.033 \\
\hline $3 \mathrm{~B}$ & 17 & Slow & $6^{\circ}$ & 0.0130 & 0.0406 & -0.00023 & 0.000002 & 0.041 & 0.039 & 0.037 & 0.035 \\
\hline $4 \mathrm{~A}$ & 12 & Fast & $0^{\circ}$ & 0.0069 & 0.0495 & -0.00191 & 0.000080 & 0.050 & 0.038 & 0.043 & 0.101 \\
\hline $4 \mathrm{~B}$ & 12 & Fast & $6^{\circ}$ & 0.0090 & 0.0440 & -0.00034 & 0.000030 & 0.044 & 0.044 & 0.049 & 0.078 \\
\hline $4 \mathrm{C}$ & 11 & Fast & $0^{\circ}$ & 0.0056 & 0.0487 & -0.00127 & 0.000061 & 0.049 & 0.042 & 0.048 & 0.096 \\
\hline $4 \mathrm{D}$ & 11 & Fast & $6^{\circ}$ & 0.0055 & 0.0418 & 0.00037 & 0.000014 & 0.042 & 0.047 & 0.055 & 0.079 \\
\hline
\end{tabular}

${ }^{\mathrm{a}} \Delta S(\lambda)$ (in $\left.\mathrm{AU}\right)=a+b \lambda$, where $\lambda$ (in deg.) is the solar $\mid$ Longitude $\mid$ of the solar source.

${ }^{\mathrm{b}} \Delta$ longitude is the $\lambda$ bias, found through the least-squares fitting.

${ }^{\mathrm{c}}$ Sigma is effectively the square root of the chi-squared value of the fit, as determined by a standard Interactive Digital Language fitting routine.

${ }^{\mathrm{d}} \Delta S(\lambda)($ in $\mathrm{AU})=a^{\prime}+b^{\prime} \lambda+c^{\prime} \lambda^{2}$.

ure 3, however, |Longitude| (which is the parameter actually shown in the plot) is formed by creating effectively a new "center line," by shifting all longitudes of the MCs' sources by various trial $\Delta$ longitudes (so new long. $=$ old long. $-\Delta$ long.), and then by taking the absolute value of this difference. We show the results specifically for $\Delta$ long. (called "shift" in Figure 3 ) for $0^{\circ}$ and $6^{\circ}$ in Figures $3 \mathrm{~A}$ and $3 \mathrm{~B}$, respectively. [We point out that many $\Delta$ long. shifts were considered. In fact, we tried ten total shifts - five $+2^{\circ}$ shifts in longitude (i.e., $+2^{\circ}, 4^{\circ}, \ldots,+10^{\circ}$ ) and five $-2^{\circ}$ shifts (i.e., $\left.-2^{\circ},-4^{\circ}, \ldots,-10^{\circ}\right)-$ and determined that the best relationship between $\Delta S$ and |Longitude| was the one given by using the $+6^{\circ}$ shift. Hence, the $+6^{\circ}$ shift will be discussed in the following, but the $0^{\circ}$ shift will be included for comparison. Space does not allow displaying results of all of the considered shifts, most of which gave undesirable results.] Notice that this center line is thought of as having a spiral curve starting at the source and ending at or near the nose of the MC. (It might have been more informative to consider solar longitude itself, with special attention to whether it was from West or East, but poor statistics forced us to fold everything to the West by using the absolute value of longitude. This is especially true for the "Fast set," as we will see later.) These data are least-squared fitted by both straight lines and quadratic curves with the associated fit coefficients given in Table 2, under the category of speed range of "Slow." There is no significant difference in the results of these two shifted sets, for either first or second order, although the case for the shift of $0^{\circ}$ (for both orders) gives slightly lower sigmas $(0.0125$ 
and $0.0128 \mathrm{AU}$, respectively), where "sigma" is effectively the same as $\sqrt{ }$ (chi-squared) for the appropriate fit. The "sigmas" are determined by a standard Interactive Digital Language fitting routine, called poly_fit. It is clear that the c.c. of -0.17 (or -0.12 ) is very poor, indicating essentially no correspondence between $\Delta S$ and |Longitude| for the slow/average speed range. This is true even though the associated MCs were fast enough for upstream conditions to generate upstream shock waves. For these slower speeds we obtain the surprising result that $\Delta S$ is typically $0.040 \pm 0.013$ AU essentially at all longitudes.

We now examine the reduced set of MCs for the fast sheath speed range $(N=12)$, where the situation is obviously very different. We again examine the data for the same two shifts of $\Delta$ long. of $0^{\circ}$ and $6^{\circ}$ and call attention to the fact that one point (which will be seen as an outlier with a very low $\Delta S$ of $0.027 \mathrm{AU}$ ) has a borderline sheath speed of $502 \mathrm{~km} \mathrm{~s}^{-1}$ for this category and will be dropped for expository reasons, giving $N=11$. Figures $4 \mathrm{~A}$ and $4 \mathrm{~B}$ show the cases of $N=12$ for shifts of $\Delta$ long. of $0^{\circ}$ and $6^{\circ}$, respectively, and Figures $4 \mathrm{C}$ and $4 \mathrm{D}$ show the cases of $N=11$ for the same shifts of $\Delta$ long. of $0^{\circ}$ and $6^{\circ}$, respectively. Table 2, for the speed range of "Fast," gives the associated fit coefficients for the first- and second-order curves. In all three cases of Figures $4 \mathrm{~A}-4 \mathrm{C}$ the minimum is a somewhat implausible position (for example, near $11^{\circ}$ for case A, whereas it is expected to be at or near $0^{\circ}$ ), and the minima are poorer for the cases of $\Delta$ long. of $0^{\circ}$. Hence, either the outlier or incorrect longitude biasing (i.e., the shift) caused the implausible distortion. Table 2, for the quadratic fit (bottom), shows that the case of $N=11$ and $\Delta$ long. $=6^{\circ}$ (corresponding to Figure 4D, quadratic fit) gives the best c.c. of 0.93, the lowest sigmas (0.0057 and $0.0055 \mathrm{AU}$ for the first- and second-order fittings, respectively), and the most plausible minimum position. Note that across the board the second-order curves have the better sigmas, except for the one corresponding to Figure 4B. In every respect the case corresponding to Figure 4D satisfies what we expect for a best case, whether we are considering the linear versus second-order fit (but again the quadratic fit has smaller sigma), behavior at $0^{\circ}$ (i.e., continuity of derivative at $0^{\circ}$ as East goes over to West), and growth in $\Delta S$ versus |Longitude|. We also point out here that, since the ecliptic-plane projection of the MC's front surface (i.e., near the MC's nose within $\pm 40^{\circ}$ ) should be reasonably well approximated by an ellipse, and the upstream shock surface well represented by a hyperbola, the difference, giving $\Delta S$, is expected to be least-squares fitted quite well by a quadratic form also - not a linear one. Obviously the first-order fit would not give a proper shape as we pass through $0^{\circ}$ (i.e., its derivative would be discontinuous for $\Delta S$ versus actual longitude at $0^{\circ}$ ). The straight-line least-squares fits were shown here mainly for edification.

It is interesting to see what values we get for $\Delta S$ at $60^{\circ}$, using the second order forms for $\Delta S$ and the coefficients in Table 2, for all four cases associated with Figures $4 \mathrm{~A}-4 \mathrm{D}$, which are $\Delta S\left(\right.$ at $\left.60^{\circ}\right)=0.223,0.132,0.192$, and $0.114 \mathrm{AU}$, respectively. If we assume that $\Delta S$ (at $\left.60^{\circ}\right)=0.114 \mathrm{AU}$ (Figure 4D) is the best result, based on arguments given here, then $\Delta S$ (at $\left.60^{\circ}\right)$ is 2.7 times the value of $\Delta S\left(\right.$ at $\left.0^{\circ}\right)(=0.042 \mathrm{AU})$; see the last line of Table 2 . One thing is clear: Regardless of which case is most acceptable, we can have confidence that for the fast set, on average, $\Delta S$ is close to $0.045 \mathrm{AU}$ at $0^{\circ}$ longitude and that $\Delta S$ grows significantly with solar longitude, as expected. We see that, even for the quadratic slow/average set, $\Delta S$ at $0^{\circ}$ (being $0.040 \mathrm{AU}$ ) is very close to this value of $0.045 \mathrm{AU}$. It is only for the larger longitudes that the estimates significantly differ between the fast and slow/average sets.

\section{Summary and Discussion}

Average sheath thickness surrounding a typical MC, as viewed in the ecliptic plane, is estimated for a "reduced" set of Wind MCs in terms of its variation with respect to longitude 

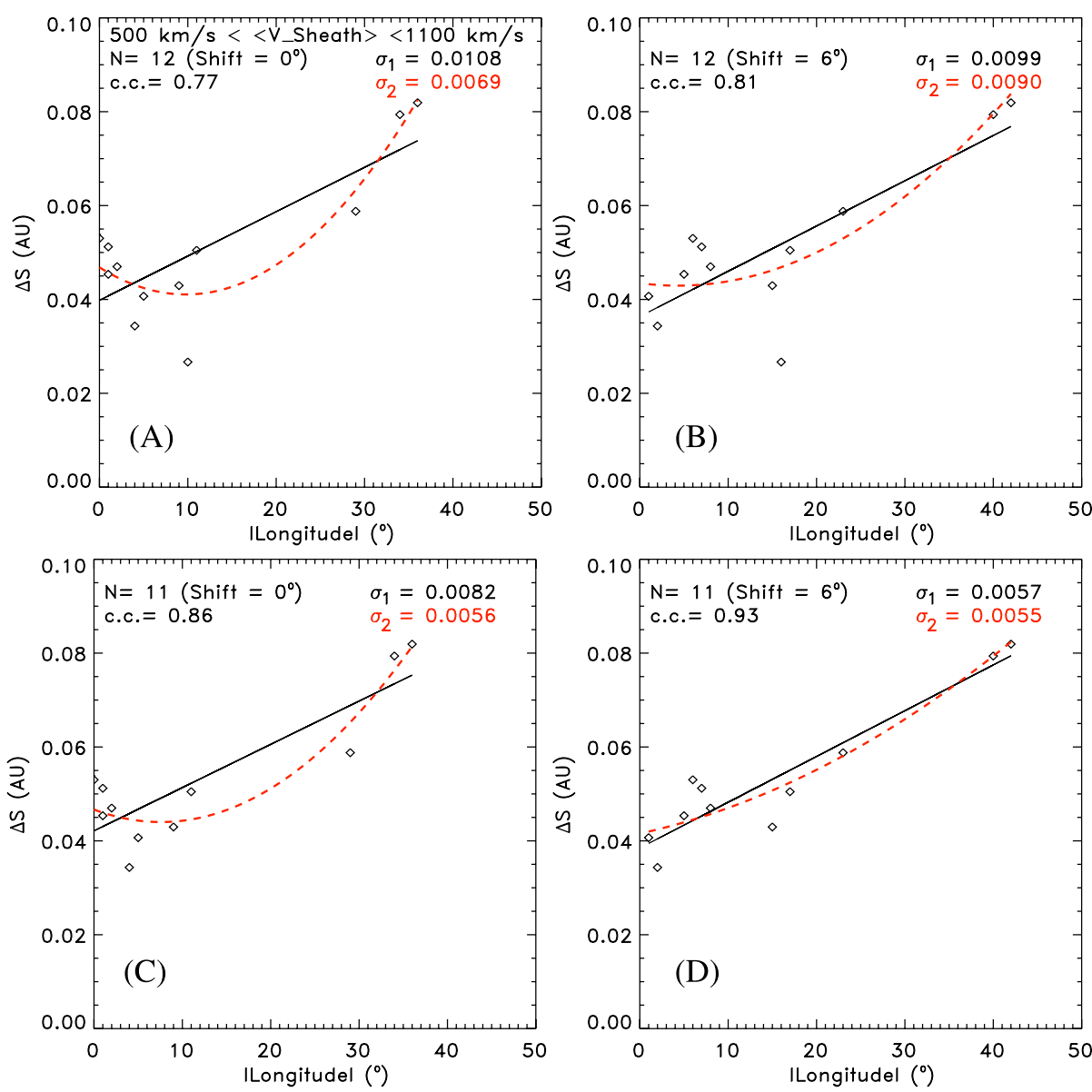

Figure 4 Sheath thickness $(\Delta S)$ versus |Longitude| of solar source for the MCs from the reduced set for the fast sheath speed range (i.e., $500-1100 \mathrm{~km} \mathrm{~s}^{-1}$ ) (Table 1, bottom); the actual observations are given as diamonds. (A) and (B) show the full $N=12$ cases for longitude shifts of $0^{\circ}$ and $6^{\circ}$, respectively. (C) and (D) show the same set of MCs, except reduced by one low-value $\Delta S(=0.027 \mathrm{AU})$, a clear outlier, again for longitude shifts of $0^{\circ}$ and $6^{\circ}$, respectively; hence, $N=11$. The quadratic least-squares fits are given as red dashed curves and the straight-line fits are given as black solid lines.

of the solar source of each MC. By reduced set we mean one that resulted from various restrictions to arrive at an ecliptic-plane view. These restrictions were $i$ ) existence of a driven upstream shock wave, ii) knowledge of the solar source (but ignoring those over $70^{\circ} \mathrm{E}$ or $\left.70^{\circ} \mathrm{W}\right)$, iii) absolute value of $\mathrm{MC}$ axial latitude $\left.\left(\left|\theta_{\mathrm{A}}\right|\right)<40^{\circ}, i v\right)$ absolute value of solar source latitude $\left(\left|\theta_{\mathrm{S}}\right|\right)<30^{\circ}$, and $v$ ) restricted closest approach distance (CA) from the $\mathrm{MC}$ axis of observing spacecraft to be $|\mathrm{CA}|<80 \%$. After obtaining this "reduced" set of $29 \mathrm{MCs}$, from the original 100 cases, we found that it was necessary to split it into two sheath speed ranges: one of low/average speeds (in the range $300-500 \mathrm{~km} \mathrm{~s}^{-1}$ ) and one of fast speeds $\left(500-1000 \mathrm{~km} \mathrm{~s}^{-1}\right)$. Without this splitting very poor linear correlation coefficients for $\Delta S$ versus |Longitude| result, but, in fact, we probably should not expect $\Delta S$ to be independent of speed range. Only the fast speed set yielded plausible results, in the sense of results looking like that pictured in Figure 1. For the Figure 4D set (where the long. 
shift is $6^{\circ}$ ), quadratic least-squares fitted estimates of $\Delta S$ go from 0.042 to 0.055 to 0.079 to $0.114 \mathrm{AU}$ (at $1 \mathrm{AU}$ ) over the solar longitudes of $0^{\circ}$ to $20^{\circ}$ to $40^{\circ}$ to $60^{\circ}$, respectively, where $0^{\circ}$ represents the adjusted center line of the average $\mathrm{MC}$, and $\Delta S\left(60^{\circ}\right)$ is an extrapolation. This estimated fit is well determined with a sigma for the quadratic fit of $0.0055 \mathrm{AU}$; see Figure 4D. Note that the quadratic fit for Figure 4B, containing the outlier (so $N=12$ ), is very similar to the Figure $4 \mathrm{D}$ results, with $\Delta S=0.044,0.049,0.078$, and $0.132 \mathrm{AU}$, again for solar longitudes of $0^{\circ}, 20^{\circ}, 40^{\circ}$, and $60^{\circ}$, respectively. For the slower speeds we obtain the surprising result that $\Delta S$ is typically more-or-less constant at $0.040 \pm 0.013 \mathrm{AU}$ at all longitudes, suggesting that the MC as driver, when moving close to the normal solar wind speed, has little influence on sheath thickness; see Figure 3.

The sigmas in Table 2 may be considered a measure of the uncertainty in $\Delta S$ based on the particular fitting procedure. There is another contributor to this uncertainty. It is based on the ability to determine the actual time of the front boundary of a MC and the shock ramp to estimate $\Delta S$ for any given case. The shock ramp time is usually determined very accurately (i.e., to seconds). The usual time uncertainty expected for front boundaries of MCs is about half an hour. This estimate is based on information applicable for Wind MC boundary times (over approximately the first nine years of the mission) determined by two methods at the Web site http://lepmfi.gsfc.nasa.gov/mfi/mag_cloud_table9.html. The time uncertainty estimated for the MCs used here was specifically $\left|\Delta t_{\mathrm{UN}}\right|=31$ minutes, from the 29-case subset of this Web listing. Specifically, this value was derived from the average (over the $N=29$ cases) of the absolute difference between a front boundary time obtained from visual inspection and that used in the Lepping, Jones, and Burlaga (1990) MC parameter-fitting procedure. If we use this 31-minute-average time uncertainty to estimate its contribution to the $\Delta S$ (average distance) uncertainty [called $\delta(\Delta S)$ here], we find the following, where a speed of $514 \mathrm{~km} \mathrm{~s}^{-1}$ (i.e., the average sheath speed from Table 1) is assumed:

$$
\delta(\Delta S)=31 \mathrm{~min} \times 514 \mathrm{~km} \mathrm{~s}^{-1}=0.0064 \mathrm{AU} .
$$

(This value may be considered a maximized estimate of uncertainty, since upon careful analysis of the front boundary time for any specific case, it should be shorter and maybe considerably so.) As we see, this value is comparable to the sigma for Figure 4D (quadratic fit) in Table 2 and smaller than most of the other sigmas in the table. Typically a MC has a diameter of $0.25 \mathrm{AU}$ (e.g., Lepping et al., 2006). Hence, this value of $0.0064 \mathrm{AU}$ is only $2.6 \%$ of a typical MC diameter, and about $15 \%$ of typical sheath thicknesses near the shock's nose, as determined here. We warn that for any given case this uncertainty may be quite different from this typical estimate. Finally, we emphasize that the $6^{\circ}$ shift, if indeed it is the best longitude shift to use, is thought of as a discovered "best-bias" value to find the "center line," and not to be thought of as an uncertainty in any sense. Therefore, it did not directly enter into the estimates of uncertainty given here.

Figure 2B shows a scatter plot, for the full $N=29 \mathrm{MCs}$, of the average sheath speed $\left\langle V_{\text {SHEATH }}\right\rangle$ versus a difference speed $(\Delta V)$, defined in the caption of the figure. It is easily seen that $\Delta V$ is not distributed into two separate modes or portions, slow and fast. Nevertheless, as we see, there is a relatively good correlation between $\left\langle V_{\text {SHEATH }}\right\rangle$ and $\Delta V$, where c.c. (linear) $=0.80$. Also, in Figure $2 \mathrm{~A}$, which is similar to Figure $2 \mathrm{~B}$, except now the relative speed $\Delta V$ is replaced by $\left\langle V_{\mathrm{MC}, \mathrm{T}}\right\rangle$ (the average $\mathrm{MC}$ speed across the full MC's duration), there is an even better linear correlation: c.c. $=0.84$. These figures indicate that the separation of our analysis according to slow and fast sheath speeds $\left(\left\langle V_{\text {SHEATH }}\right\rangle \mathrm{s}\right)$ is essentially also a separation of the analysis according to slow and fast $\Delta V \mathrm{~s}$, or slow and fast $\left\langle V_{\mathrm{MC}, \mathrm{T}}\right\rangle \mathrm{s}$, to good approximations, because of the high correlations. Therefore, our Figure 4 (special case 
of $N=11$ and shifts of $6^{\circ}$ ) holds approximately for the faster $\Delta V \mathrm{~s}$, that is, for the MCs that are faster relative to the upstream solar wind speed, or hold simply for the faster MCs.

This study suffers from having low statistics, especially for the fast speed set at higher longitudes and around solar longitude of $20^{\circ} \pm 5^{\circ}$, where there is a gap, which is obviously mapped to larger values (i.e., to near $32^{\circ}$, for the $6^{\circ}$-shifted estimates - see panels B and D of Figure 4). Perhaps when a larger set of MCs is developed with associated shocks and known sources, under our restrictions, we can get the functional form of $\Delta S$ with greater accuracy, and, more important, with no need for folding East and West solar longitudes together. In any case, we can be relatively sure that fitting $\Delta S$ will still yield a second-order relationship, as we have found here. Also the sheath thickness should be expected to depend on the kind of shock (parallel, perpendicular, or oblique) that exists in the neighborhood of the sheath being considered; this separate categorization was not possible here. And likewise upstream Mach number should play a role in helping to determine sheath thickness and similarly for the specific shape of the nose of the MC (e.g., Russell and Mulligan, 2002a). For example, we know that for an object of needle-nose shape driving a shock, the shock surface would approximately touch the object at the nose and globally the shock surface appears coneshaped. The $\Delta S$ for this case would surely be different from the case resulting from a fast sphere moving through a plasma and driving a shock. We do not have access to such information ("type" or Mach number) for more than one longitude (the one where the spacecraft measurements were made), to account for any such variation of $\Delta S$ with longitude, and we have virtually no confident information on global shape. We also point out that MCs are not always ejected or transported radially from the Sun (see, e.g., Webb et al., 2000, on the 15 May 1997 event, and Berdichevsky et al., 2005, on the 20 March 2003 event, which are examples of such nonradially moving MCs), as tacitly assumed here. Nonradial transport is most likely to be the case for the slower MCs, apparently consistent with what we find (i.e., a greater scatter of $\Delta S$ with solar source longitude for the slower MCs and essentially no obvious relationship between $\Delta S$ and $\lambda$ ). In fact, this complication of nonradial transport is probably responsible for some of the scatter of points in both Figures 3 and 4. Apparently, at this time, we have to be satisfied with the limited results shown in these figures (and in Table 2): We have estimated relationships that approximately typify average sheath thickness at $1 \mathrm{AU}$; that is, it is approximately constant for all longitudes for slow MCs (i.e., $\langle\Delta S\rangle \approx 0.04 \mathrm{AU})$, but it is a quadratic function of solar longitude for fast MCs, being close to the slow MCs' value near the nose (i.e., where $\langle\Delta S\rangle$ (fast) is $0.042 \mathrm{AU}$ ). Russell and Mulligan (2002a) point out that a sheath thickness of about 0.025 AU should be expected when based on estimates of typical ICME half-thicknesses, but they suggest that empirical evidence indicates values for sheath thickness closer to $0.1 \mathrm{AU}$. We do not find values as large as $0.1 \mathrm{AU}$, from this statistical study, except for large $\lambda \mathrm{s}$, but interestingly our average value of $\langle\Delta S\rangle \approx 0.04 \mathrm{AU}$ for fast and slow cases at and near the MC's nose is close to the middle of the two estimates given by Russell and Mulligan (2002a).

If we accept the coefficients for the last line in Table 2, corresponding to Figure 4D, as marginally the best for describing the second-order relationship between $\Delta S$ and $\lambda^{\prime}$ (where $\lambda^{\prime}$ is the shifted longitude, by $6^{\circ}$ in this case) for fast cases, then explicitly $\Delta S$ is estimated by

$$
\Delta S(\text { in } \mathrm{AU})=0.0418+0.00037 \lambda^{\prime}+0.000014\left(\lambda^{\prime}\right)^{2} \quad\left(\text { where } \lambda^{\prime} \text { is in degrees }\right) .
$$

Inverting this to solve for $\lambda^{\prime}$ (and noting that $\lambda^{\prime}=\lambda_{\mathrm{OBS}}-6^{\circ}$ ) gives the actually observed source longitude as

$$
\lambda_{\mathrm{OBS}}=-7.2^{\circ}+\sqrt{\left(174 \mathrm{deg}^{2}+L\right)},
$$


where $L \equiv(\Delta S-0.0418) / 0.000014$ (in $\left.\mathrm{deg}^{2}\right)$ and where $\Delta S$ is in AU. (Note that, because $\lambda_{\mathrm{OBS}}$ in this formulation must be positive, then the sign in front of the square root must be chosen to be positive.) Besides giving some general idea of how $\Delta S$ varies with $\lambda_{\mathrm{OBS}}$ on average, as one more element in understanding the evolution of MC and upstream structures, expression (4) may be useful, for most normal cases, when trying to decide between the longitudes of two candidate solar source locations of a fast MC (or any fast ejecta) at $1 \mathrm{AU}$ by asking whether one source is more plausible than the other. In particular, the choice might be facilitated by noting the value of the observed sheath thickness $(\Delta S)$, using expression (4) to find the estimated $\lambda_{\mathrm{OBS}}$ and the quadrant of the MC's axis, and finally making a comparison with the two solar-source candidates. The MC's axis and its polarity are also needed to help in separating $\lambda$-East from $\lambda$-West, as well as being useful in their own right in helping to differentiate the solar source, as has been done often in the past. We stress that it is not likely that expression (4) will be very useful, if the two candidate solar longitudes are very close together.

Finally, Gopalswamy (2006; his Figure 2) points out that, according to the specific path that a spacecraft takes when passing through or near a MC, six possible scenarios can occur depending on whether the MC has an upstream shock wave and on the relative longitude of the path. For example, for the case of an existing upstream shock, the spacecraft may pass through only the shock and miss the MC (at large longitudes with respect to the "center" longitude), or it may pass through the shock and MC with a thick intervening sheath far off the "nose" of the MC, or it may pass through the shock and MC with a much thinner sheath at the nose. We have tried here to quantify, on average, how these values of sheath thickness are expected to vary with relative longitude.

Acknowledgements We thank Dr. Adam Szabo and Prof. Franco Mariani for Wind/MFI data handling and calibration and Tom Narock for general assistance over the past few years on this and related magnetic cloud studies. We thank Dr. Keith Ogilvie and the Wind/SWE team for use of sheath plasma speeds in calculating sheath thickness and the referee for a suggestion that led to the results presented in Figure $2 \mathrm{~B}$ and for pointing out some important references. This work was supported by the NASA Heliophysics Guest Investigator Program.

\section{References}

Badruddin, M., Venkatesan, D., Zhu, B.Y.: 1991, Solar Phys. 134, 203 - 209.

Berdichevsky, D.B., Richardson, I.G., Lepping, R.P., Martin, S.F.: 2005, J. Geophys. Res. 110, A09105. doi:10.1029/2004JA010662.

Burlaga, L.F.: 1988, J. Geophys. Res. 93, 7217-7224.

Burlaga, L.F.: 1995, Interplanetary Magnetohydrodynamics, Oxford Univ. Press, New York, 89-114.

Burlaga, L.F., Sittler, E.C. Jr., Mariani, F., Schwenn, R.: 1981, J. Geophys. Res. 86, 6673-6684.

Fairfield, D.H.: 1971, J. Geophys. Res. 76, 6700-6616.

Gopalswamy, N.: 2006, Space Sci. Rev. 124, 145 - 168.

Gopalswamy, N., Akiyama, S., Yashiro, S., Michalek, G., Lepping, R.P.: 2007, J. Atmos. Space-Terr. Phys. 70(2-4), 245-253. doi:10.1016/j.jastp.2007.08.070.

Gosling, J.T., McComas, D.J.: 1987, Geophys. Res. Lett. 14, 355 - 358.

Klein, L., Burlaga, L.F.: 1982, J. Geophys. Res. 87, $613-624$.

Lepping, R.P., Berdichevsky, D.: 2000, Recent Res. Devel. Geophys. 3, 77 - 96.

Lepping, R.P., Jones, J.A., Burlaga, L.F.: 1990, J. Geophys. Res. 95, 11957-11965.

Lepping, R.P., Wu, C.-C., Berdichevsky, D.B.: 2005, Ann. Geophys. 23, 2687-2704. Sref-ID: $1432-$ 0576/ag/2005-23-2687.

Lepping, R.P., Burlaga, L.F., Tsurutani, B.T., Ogilvie, K.W., Lazarus, A.J., Evans, D.S., Klein, L.W.: 1991, J. Geophys. Res. 96, $9425-9438$.

Lepping, R.P., Berdichevsky, D., Szabo, A., Lazarus, A.J., Thompson, B.J.: 2002. In: Lyu, L.-H. (ed.) Space Weather Study Using Multipoint Techniques, Proceedings of the COSPAR Colloquium in Pacific Green Bay, Taiwan, 27-29 September 2000, Pergamon, Oxford, 87-96. 
Lepping, R.P., Berdichevsky, D.B., Wu, C.-C., Szabo, A., Narock, T., Mariani, F., Lazarus, A.J., Quivers, A.: 2006, Ann. Geophys. 24(\#1), 215 -245. Sref-ID: 1432-0576/ag/2006-24-215.

Liu, Y., Richardson, J.D., Belcher, J.W., Kasper, J.C., Skoug, R.M.: 2006a, J. Geophys. Res. 111, A09108. doi:10.1029/2006JA011723.

Liu, Y., Richardson, J.D., Belcher, J.W., Wang, C., Hu, Q., Kasper, J.C.: 2006b, J. Geophys. Res. 111, A12S03. doi:10.1029/2006JA011890.

Marubashi, K.: 1986, Adv. Space Res. 6(6), 335-338.

Marubashi, K.: 1997, In: Crooker, N., Joselyn, J., Feynman, J. (eds.) Geophys. Monogr. Ser. 99, AGU, Washington, $147-156$.

McComas, D.J., Gosling, J.T., Winterhalter, D., Smith, E.J.: 1988, J. Geophys. Res. 93, $2519-2526$.

Morrison, P.: 1956, Phys. Rev. 101(\#4), 1397-1404.

Owens, M.J., Cargill, P.J., Pagel, C., Siscoe, G.L., Crooker, N.U.: 2005, J. Geophys. Res. 110, A01105. doi:10.1029/2004JA010814.

Riley, P., Crooker, N.U.: 2004, Astrophys. J. 600, 1035 - 1042.

Spreiter, J.R.: 1975, In: Ness, N.F. (ed.) NASA-Goddard Space Flight Center, Internal Document, Moscow, USSR, Nov. 17-21, 1975, NASA-SP-397, 135-149.

Spreiter, J.R., Summers, A.L., Alksne, A.Y.: 1966, Planet. Space Sci. 14, 223 - 253.

Russell, C.T., Mulligan, T.: 2002a, Planet. Space Sci. 50, 527-534.

Russell, C.T., Mulligan, T.: 2002b, Adv. Space Res. 29(\#3), 301-306.

Webb, D.F., Lepping, R.P., Burlaga, L.F., DeForest, C.E., Larson, D.E., Martin, S.F., Plunkett, S.P., Rust, D.M.: 2000, J. Geophys. Res. 105, $27251-27259$.

Wolfe, J.H.: 1972, In: Solar Wind 1, Asilomar Conference, March 21 -26, 1971, 170 - 197. 